\title{
Multispectral Imaging of Organ Viability during Uterine Transplantation Surgery
}

\author{
Neil T. Clancy*a,b, Srdjan Saso ${ }^{c}$, Danail Stoyanov ${ }^{\mathrm{d}}$, Vincent Sauvage ${ }^{\mathrm{a}, \mathrm{b}}$, David J. Corless ${ }^{\mathrm{e}}$, Michael \\ Boyd $^{\mathrm{f}}$, David E. Noakes ${ }^{\mathrm{f}}$, Meen-Yau Thum ${ }^{\mathrm{g}}$, Sadaf Ghaem-Maghami ${ }^{\mathrm{h}}$, J. Richard Smith ${ }^{\mathrm{h}}$, Daniel S. \\ Elson $^{\mathrm{a}, \mathrm{b}}$ \\ ${ }^{a}$ Hamlyn Centre for Robotic Surgery, Institute of Global Health Innovation, Imperial College

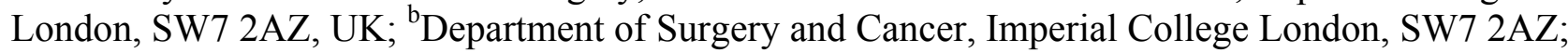 \\ ${ }^{\mathrm{c}}$ Institute of Reproductive and Developmental Biology, Hammersmith Hospital, Imperial College \\ London, W12 0NN, UK; ${ }^{\mathrm{d} C e n t r e ~ f o r ~ M e d i c a l ~ I m a g e ~ C o m p u t i n g, ~ D e p a r t m e n t ~ o f ~ C o m p u t e r ~ S c i e n c e, ~}$ \\ University College London, WC1E 6BT, UK; ${ }^{\mathrm{e}}$ Department of Surgery, Leighton Hospital, Crewe, \\ UK; ${ }^{\mathrm{f}}$ Royal Veterinary College, London, UK; ${ }^{\mathrm{g}}$ The Lister Hospital, Chelsea Bridge Road, London, \\ SW1W 8RH, UK; ${ }^{\mathrm{h}}$ Gynaecological Oncology, West London Gynaecological Cancer Centre, \\ Hammersmith Hospital, Imperial College London, W12 0NN, UK.
}

\begin{abstract}
Uterine transplantation surgery has been proposed as a treatment for permanent absolute uterine factor infertility (AUFI) in the case of loss of the uterus. Due to the complexity of the vasculature correct reanastomosis of the blood supply during transplantation surgery is a crucial step to ensure reperfusion and viability of the organ.

While techniques such as fluorescent dye imaging have been proposed to visualise perfusion there is no gold standard for intraoperative visualisation of tissue oxygenation. In this paper results from a liquid crystal tuneable filter (LCTF)-based multispectral imaging (MSI) laparoscope are described. The system was used to monitor uterine oxygen saturation $\left(\mathrm{SaO}_{2}\right)$ before and after transplantation. Results from surgeries on two animal models (rabbits and sheep) are presented.

A feature-based registration algorithm was used to correct for misalignment induced by breathing or peristalsis in the tissues of interest prior to analysis. An absorption spectrum was calculated at each spatial pixel location using reflectance data from a reference standard, and the relative contributions from oxy- and deoxyhaemoglobin were calculated using a least squares regression algorithm with non-negativity constraints.

Results acquired during animal surgeries show that cornual oxygenation changes are consistent with those observed in point measurements taken using a pulse oximeter, showing reduced $\mathrm{SaO}_{2}$ following reanastomosis. Values obtained using the MSI laparoscope were lower than those taken with the pulse oximeter, which may be due to the latter's use of the pulsatile arterial blood signal. Future work incorporating immunological test results will help to correlate $\mathrm{SaO}_{2}$ levels with surgical outcomes.
\end{abstract}

Keywords: Multispectral imaging, oxygen saturation, surgical imaging, uterine transplantation.

\section{INTRODUCTION}

Hyperspectral (HSI) and multispectral imaging (MSI) have seen widespread use in remote sensing and machine vision but are now seeing significant uptake in biomedical applications. This paper explores the use of MSI in transplant surgery to provide a measure of tissue oxygen saturation $\left(\mathrm{SaO}_{2}\right)$ to the surgeon, which is an important predictor of the viability of the organ.

*n.clancy@imperial.ac.uk; phone 4420759 41707; www.imperial.ac.uk/people/n.clancy

Advanced Biomedical and Clinical Diagnostic and Surgical Guidance Systems XII, edited by

Tuan Vo-Dinh, Anita Mahadevan-Jansen, Warren S. Grundfest, Proc. of SPIE Vol. 8935,

893510 @ 2014 SPIE · CCC code: 1605-7422/14/\$18 · doi: 10.1117/12.2040518

Proc. of SPIE Vol. $8935893510-1$ 


\subsection{Optical imaging of perfusion and ischaemia}

The health of the blood supply to an organ and its oxygen saturation are important indicators of its viability. In surgery this is typically assessed qualitatively by the surgeon using visual cues such as the colour. However, subtle colour changes associated with poor perfusion or the onset of ischaemia may be difficult to determine, particularly at their borders with healthy tissue. Optical techniques using exogenous fluorescent agents have been proposed to monitor perfusion ${ }^{1}$ but these require injection of a dye and the use of additional specialist excitation light, fluorescence filters and a sensitive, low-noise camera.

Spectral imaging techniques have been reported for the quantification of a number of chromophores such as melanin, water $^{2}$ and haemoglobin ${ }^{3-5}$. The signal from the latter is dominant in the internal anatomy and is exploited by commercially available narrowband imaging instruments in flexible endoscopy ${ }^{6-8}$. Spectral imaging techniques have yet to be commercially adopted in the surgical environment for quantitative imaging of tissue oxygenation. However, there are several systems reported in the literature that have shown the feasibility of measuring changes in oxygen saturation in tissues such as the $\operatorname{skin}^{9}$, retina ${ }^{10}$, brain ${ }^{11}$ and kidney ${ }^{12}$. Spectral imaging has also been used to investigate the effects of cold and warm ischaemia-reperfusion injury ${ }^{13}$.

In this paper we describe the use of a laparoscopic multispectral imaging system ${ }^{14}$ to follow changes in uterine oxygen saturation during transplant surgery. The system is a sequential imager using a liquid crystal tuneable filter (LCTF) optimised for the visible spectrum with a broadband white light source for illumination. The system is capable of providing high spatial resolution images of oxygen saturation and blood volume, and is compatible with open or minimally invasive procedures. Results obtained from surgeries in a rabbit and a sheep are compared with pulse oximeter readings.

\subsection{Uterine transplantation surgery}

The inability to experience a pregnancy, give birth and bring up a child because of infertility has the capacity to have a severely detrimental effect on the quality of life of a woman or couple ${ }^{15}$. Absolute uterine factor infertility (AUFI) may be congenital (Mullerian duct anomalies) or acquired (intrauterine adhesions or premenopausal hysterectomy) and renders women infertile ${ }^{16}$. However, uterine transplantation surgery (UTn) is a potential benefit for cases where uterusloss results in AUFI. Recent results in animal models ${ }^{17}$ have shown the development of the surgical techniques to allow the first surgeries in humans.

Correct reconnection (anastomosis) of the blood supply in the transplanted organ is essential to allow reperfusion and ensure that the organ survives. However, injuries relating to reperfusion following periods of 'warm' (vascular clamping) or 'cold ischaemia' (while preserved outside the body after retrieval) are a major problem. The resulting damage to the parenchyma and the microcirculation during these periods is called ischemia-reperfusion injury ${ }^{16}$.

Reliable quantitative monitoring of the blood supply to tissue in the operating theatre remains challenging. Previous work has shown that by clamping a pulse oximeter probe directly onto the organ it is possible to measure oxygen saturation and 'perfusion index' (a relative measurement of blood volume) ${ }^{18}$. However, this only reflects arterial oxygenation and gives no spatial information on the organ's oxygen supply as a whole. Additionally, the need for direct contact with the tissue means that positioning the probe is a practically challenging task.

\section{MATERIALS AND METHODOLOGY}

\subsection{Multispectral imaging laparoscope}

A laparoscopic multispectral imaging system has been developed and used in a previous pilot study ${ }^{14}$, and is detailed in Fig. 1. A $30^{\circ}$ laparoscope (Karl Storz $\mathrm{GmbH}$, Tuttlingen, Germany) was attached to a liquid crystal tuneable filter (LCTF; Varispec, Cri, Inc., USA) and monochrome camera (DCU223M, Thorlabs Ltd., UK). Visible light from a xenon light source (xenon 300, Karl Storz GmbH, Tuttlingen, Germany) was coupled into the laparoscope's illumination channel using a fibre optic light cable. For a single measurement the LCTF was scanned from 500-620 nm in steps of $10 \mathrm{~nm}$ resulting in a data cube of 13 images. Reference measurements of a standard white reflectance target (Spectralon, Labsphere, Inc., USA) were made prior to the clinical study and used to correct for the spectral shape of the xenon light's output and for the wavelength-dependent transmission and sensitivity characteristics of the MSI system. 

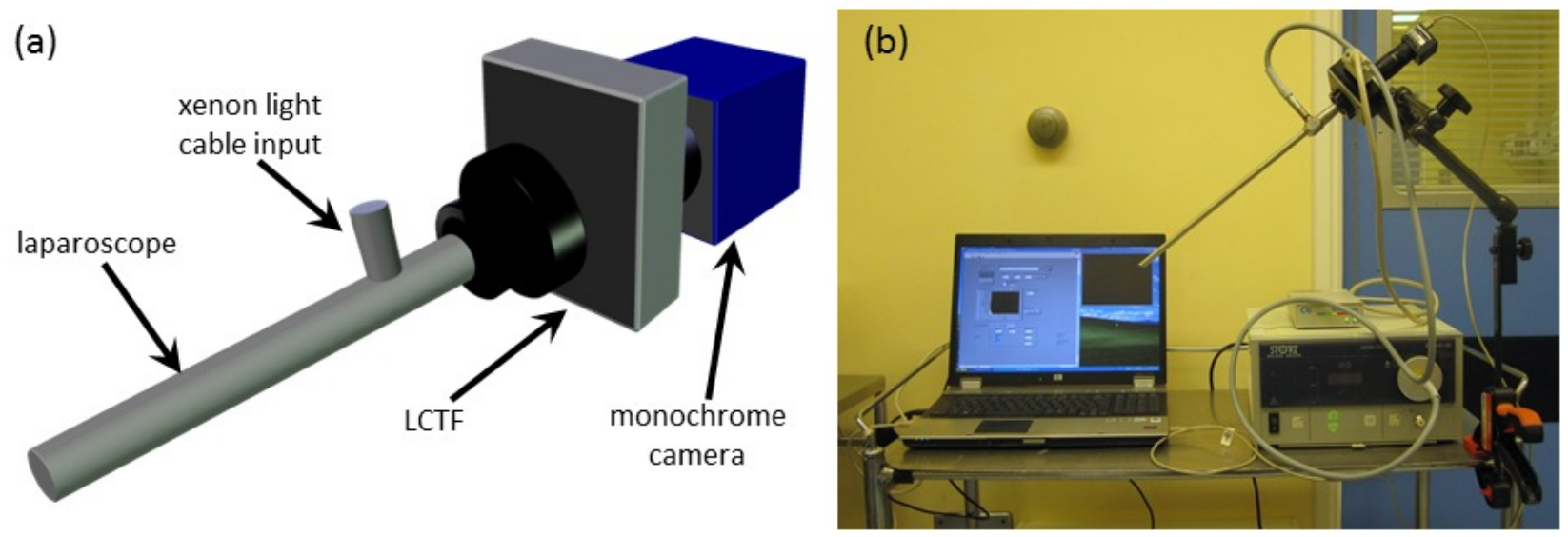

Figure 1. Multispectral imaging laparoscope. (a) System schematic showing laparoscope, LCTF, camera and xenon light source. (b) Photograph of clinical system in the operating theatre.

In order to estimate the oxygen saturation at a particular position in the tissue it is necessary to make a number of assumptions: that oxy and deoxyhaemoglobin are the only optical absorbers in the field-of-view, that scattering is constant across the wavelength range being examined and that the penetration depth of light across the wavelength range of interest is approximately constant. Taking this into account the measured reflectance spectrum at any point can be considered as a linear combination of both haemoglobin species, with an additional additive term to account for losses due to scattering. Therefore the experimentally-measured and theoretically-predicted absorbance at a particular spatial pixel are given by Eq. 1:

$$
A(\lambda)=-\ln \left(\frac{I(\lambda)-I_{D}(\lambda)}{I_{R}(\lambda)-I_{D}(\lambda)}\right) ; A(\lambda)=\left[H b O_{2}\right] \varepsilon^{H b O_{2}}(\lambda)+[H b] \varepsilon^{H b}(\lambda)+\alpha
$$

where $A$ is absorbance, $\lambda$ is wavelength, $I, I_{R}$ and $I_{D}$ are the reflected light intensities from the sample, reflectance standard and dark current respectively, $\left[\mathrm{HbO}_{2}\right]$ and $[\mathrm{Hb}]$ are concentrations of oxy and deoxyhaemoglobin respectively, $\varepsilon$ is the known molar extinction coefficient of the chromophores and $\alpha$ is a constant to account for light attenuation by other mechanisms, such as scattering. Linear least-squares regression was used to calculate the values for the three unknown parameters, $\left[\mathrm{HbO}_{2}\right],[\mathrm{Hb}]$ and $\alpha$, using known values of the extinction coefficients of haemoglobin ${ }^{19}$ and the measured absorbance at each pixel. From these values Eq. 2 was used to calculate total haemoglobin ( $H b t)$, which is indicative of blood volume, and oxygen saturation $\left(\mathrm{SaO}_{2}\right)$ :

$$
\mathrm{Hbt}=\mathrm{HbO}_{2}+\mathrm{Hb} ; \mathrm{SaO}_{2}=\frac{\mathrm{HbO}_{2}}{\mathrm{Hbt}} \times 100
$$

The possibility of using a metric of blood volume compatible with standard RGB cameras was also investigated using Eq. 3. This is a ratiometric measure that uses the intensity variation between the red and green regions of the spectrum as an indicator of light absorption ( $A b s)$ by blood. Integrating the LCTF data across the appropriate wavelengths and weighting the absorption by the measured RGB sensitivity characteristics of a commercial camera allowed calculation of total intensity in the red $\left(I_{\text {Red }}\right)$ and green $\left(I_{\text {Green }}\right)$ regions ${ }^{20}$.

$$
A b s=\frac{I_{\operatorname{Re} d}-I_{\text {Green }}}{I_{\operatorname{Re} d}}
$$

The error at each pixel was computed as the coefficient of determination $\left(\mathrm{r}^{2}\right.$ value $)$ to provide a measure of the fit of the model to the experimental data. Pixels with $\mathrm{r}^{2}$ less than 0.9 were discarded. In order to display the processed $\mathrm{SaO}_{2}$ image, the data were superimposed on a colour image of the tissue reconstructed from the multispectral stack ${ }^{20}$ in a transparent layer weighted by the normalised $H b t$ image. 


\subsection{Image registration}

Motion during image acquisition, due to breathing and peristalsis, had the effect of causing misalignment in the data cube. This appeared as blur in the reconstructed colour images and low $\mathrm{r}^{2}$ pixels in the $\mathrm{SaO}_{2}$ images. A feature-based registration algorithm has been developed to reduce these effects ${ }^{11}, 14$. This employs a sparse feature detection scheme in a triangular mesh and tracking of a set of salient features from one wavelength to the next. The mesh is then warped to align the detected features and register the images. The solution is propagated through the entire image stack, reducing the problem of wavelength-dependent visibility of certain objects, such as small blood vessels. The effect of the registration algorithm is shown in Fig. 2 using colour images reconstructed from data from rabbit and sheep experiments. The raw images show the effect of large translational movements resulting in vessel misalignment (Fig. 2 (a), centre of image), 'blur' (Fig. 2 (b)) and peristalsis resulting in deformation of the cornuas (Fig. 2 (c)). After registration however, a significant improvement is noted with alignment of the vessels (Fig. 2 (d)), reduction of blur (Fig. 2 (e)) and improved definition of the cornual structures (Fig. 2 (f)), despite severe peristaltic deformation. Note that the position of specular highlights depends on the tissue surface normal direction and these move differently to the surface features. This means that colour artefacts from the specular highlights remain in the registered images, but in any case these regions do not contain useful spectroscopic information and can be ignored.
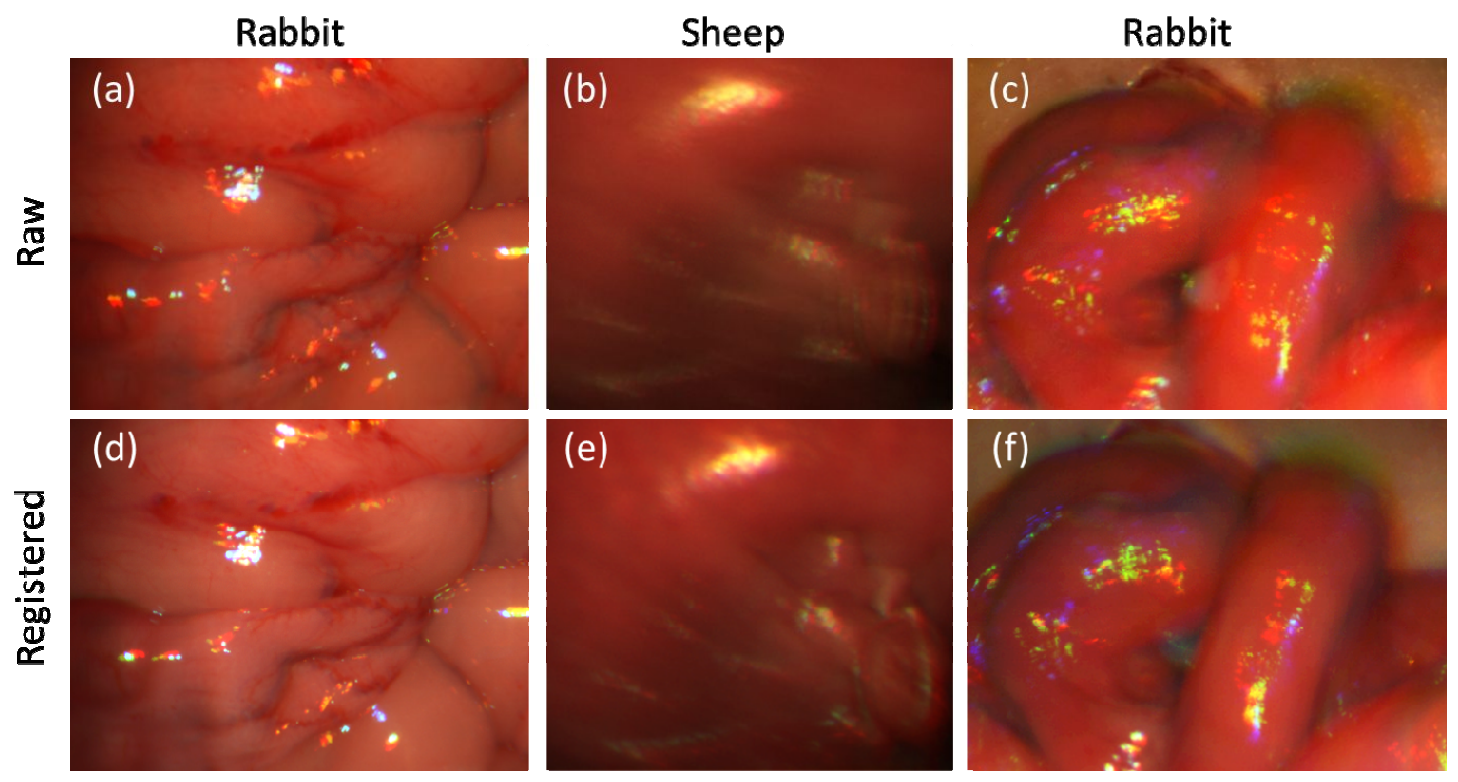

Figure 2. MSI registration. Colour images of rabbit and sheep uterus reconstructed from the multispectral data cube. (a-c) Motion during the acquisition is manifested as blur in the uncorrected images. (d-f) Motion-corrected images.

\subsection{Intraoperative imaging}

Oxygenation of the uterus was investigated during two UTn surgeries: a rabbit $(\sim 4 \mathrm{~kg})$ and a sheep $(\sim 60 \mathrm{~kg})$. Rabbit UTn was conducted between two animals (allotransplant procedure), with the recipient animal recovered following surgery, while the sheep UTn was conducted as an autotransplant under terminal anaesthesia. The surgical procedure involved removal of the uterus with an aortic-caval microvascular patch. The graft was then implanted with anastomosis of its aorta and vena cava to those of the recipient, along with reattachment of the cornuas and vagina to ensure correct positioning and support. All animal experiments were conducted under a Home Office approved licence. 

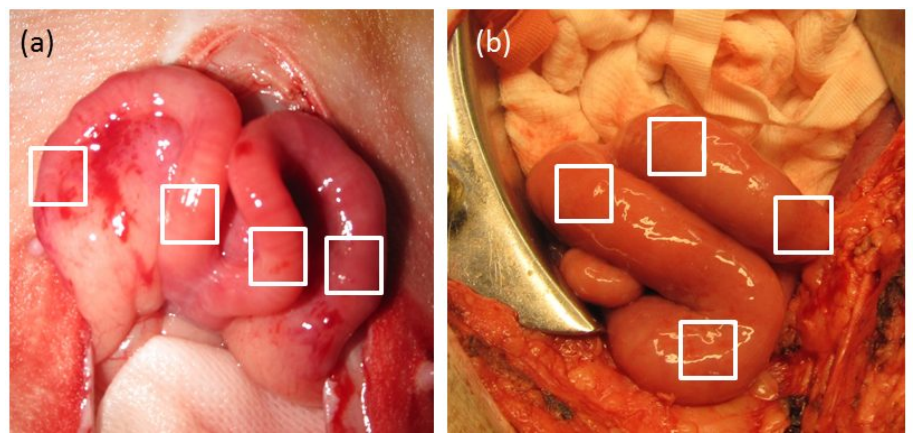

Figure 3. Photograph showing the uterus anatomy and pulse oximetry measurement points in the (a) rabbit and (b) sheep.

Oxygenation measurements were made immediately after exposure of the uterus in the donor, prior to retrieval, and after completion of the vascular anastomoses in the recipient. In the case of the rabbit, the recipient's native uterus was also measured before a hysterectomy was carried out in preparation for receipt of the donor organ. Measurements of $\mathrm{SpO}_{2}$ using a pulse oximeter (Datex-Ohmeda 3600P; Datex-Ohmeda, Colorado, USA) were made by clamping the probe, with the aid of a sterile camera bag, onto the organ and recording the oxygen saturation at the medial and lateral aspects of the right and left cornuas (Fig. 3). MSI was then carried out, including acquisition of a background signal $\left(I_{D}\right)$, with the xenon light switched off, to correct for any ambient light detected by the system. Regions of interest in the $\mathrm{SaO}_{2}$ images, corresponding approximately to the pulse oximeter measurement sites, were selected and used to calculate average saturation values.

\section{RESULTS}

Colour images of the rabbit uterus reconstructed from the MSI stack and the resultant $\mathrm{SaO}_{2}$ images are shown in Fig. 4 before and after UTn. The well-perfused, bright pink/red colour of the organ in its native state (Fig. 4 (a)) reflects its high oxygenation, which is visible in the composite image (Fig. 4 (b)). Specular highlights, visible as areas of saturated pixels in the colour images, are automatically excluded in the composite image due to their low $\mathrm{r}^{2}$ scores. The transplanted organ, imaged straight after reanastomosis of the graft, is seen to be partially reperfused almost immediately. However, its appearance is still noticeably more pale than that of the native condition (Fig. 4 (c)). This initial low perfusion is seen in the processed image as regions of lower $\mathrm{SaO}_{2}$ and areas of semi-transparency in the pseudocolour layer (Fig. 4 (d)).
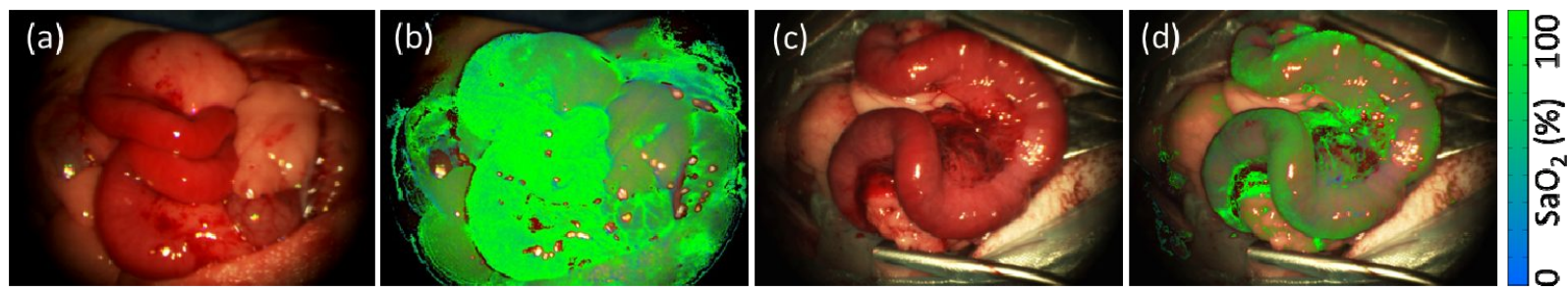

Figure 4. Colour and composite oxygenation images of the rabbit uterus in the donor (a,b) and recipient (c, d) animals. The $\mathrm{SaO}_{2}$ is overlaid on the reconstructed colour image in a pseudocoloured layer with its transparency weighted by the $\mathrm{Hbt}$ image.

Average $\mathrm{SaO}_{2}$ was calculated from regions of interest in the cornuas, as described in Section 2.3, during UTn on a rabbit and a sheep. The average saturation of each region of interest in each animal before and after UTn is shown in Fig. 5. In both animals a drop in organ oxygenation is seen by the pulse oximeter $\left(\mathrm{SpO}_{2}\right)$ and MSI $\left(\mathrm{SaO}_{2}\right)$ systems posttransplantation, although it is more pronounced in the rabbits. The oxygenation measured by MSI is also consistently lower than that of the pulse oximeter. 


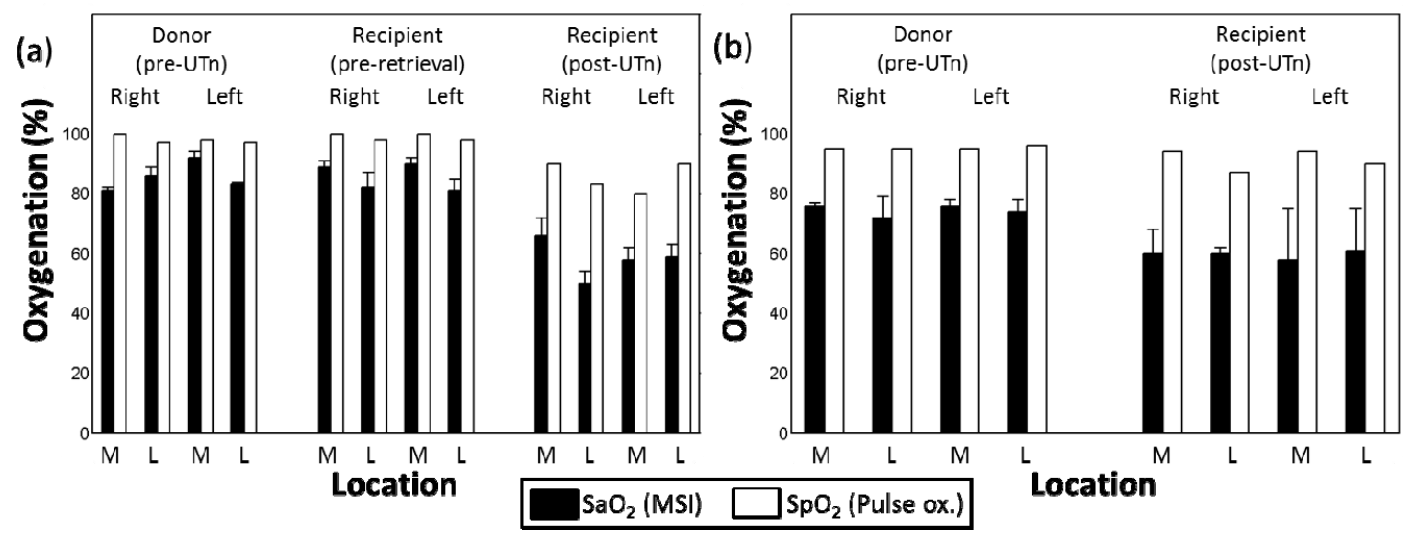

Figure 5. Variation of cornual oxygenation as measured by MSI $\left(\mathrm{SaO}_{2}\right)$ and pulse oximetry $\left(\mathrm{SpO}_{2}\right)$, before and after surgery, in (a) the rabbit and (b) the sheep at medial (M) and lateral (L) locations on the left and right sides of the uterus. The error bars represent the variation over four successive MSI measurements. Only one pulse oximetry measurement was made at each location.

Blood volume was calculated separately using Eq. 2 and Eq. 3 in order to investigate the relationship between total haemoglobin and the signal from the red and green colour planes reconstructed from the multispectral datacube. The results for the rabbit uterus are compared qualitatively and quantitatively in Fig. 6.
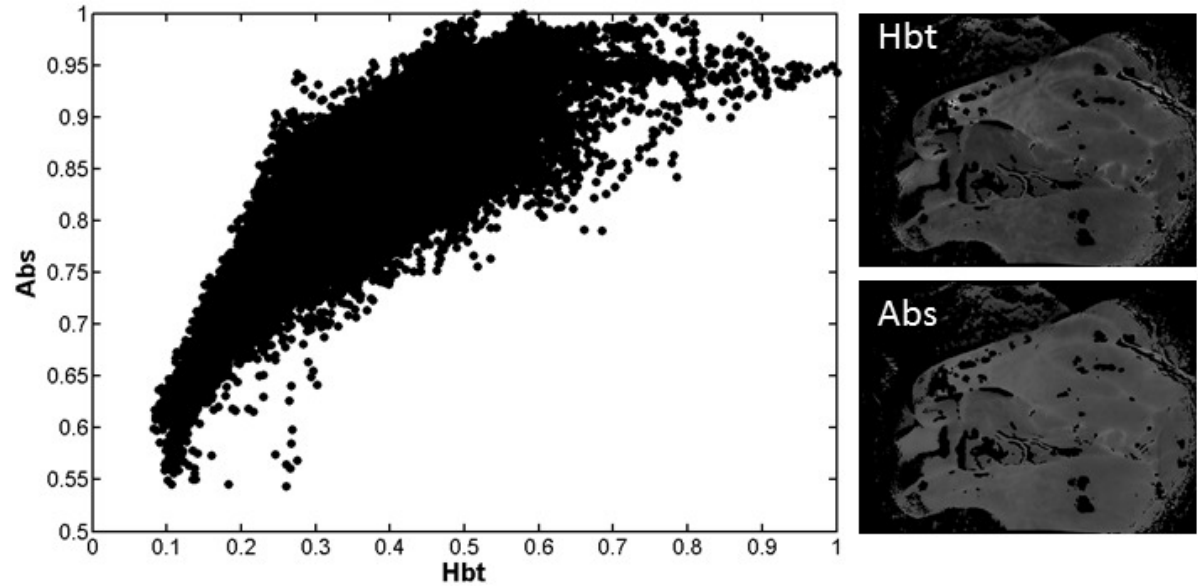

Figure 6. Comparison of total haemoglobin $(\mathrm{Hbt})$ and the blood volume metric $\mathrm{Abs}$. The scatter plot shows a pixelwise comparison of the intensity values in the corresponding greyscale images on the right and indicates a correlation between the two variables.

Both intensity images in Fig. 6 have broadly similar characteristics, with corresponding areas having approximately equal variations in brightness. A pixelwise comparison across the images, although noisy, shows a strong proportionality between the two. Calculation of the Pearson correlation coefficient between $H b t$ and $A b s$ yields a value of 0.81 .

\section{DISCUSSION}

Both MSI and pulse oximetry were able to confirm reestablishment of perfusion and oxygen supply to the organ following reanastomosis of the blood supply in the transplant surgeries monitored. Blood volume was indicated by total haemoglobin, which was shown to have a proportional relationship with the $A b s$ parameter. This is due to the fact that integrating the reflected intensities over the red and green wavelengths averages out the spectral differences between the haemoglobin species and makes the signal reflective of $\mathrm{Hbt}$ instead.

The transplant procedure in the rabbit and sheep subjects was carried out under sterile conditions meaning that the pulse oximeter, as a contact measurement, had to be shielded using a protective bag. This increased the practical difficulty of the measurement for the surgeon as manipulating the organ inside the jaws of the probe and obtaining a reliable 
plethysmograph at four different sites proved to be time-consuming. The MSI system, on the other hand, had the advantage of being non-contact and obtaining data on the entire organ during one acquisition.

The main disadvantage of the current MSI system is its operating speed, as each acquisition takes on the order of 15 seconds, followed by offline registration and processing of the data cube. Significant misalignment of the image stack due to motion and deformation of the tissue can occur in this time frame, though this was reduced using the featurebased registration software discussed in Section 2.2. Errors due to complex deformations however, such as those due to large peristaltic motion shown in Fig. 2, could not be fully eliminated.

Both MSI and pulse oximetry detected a drop in oxygenation following UTn. This may be due to the fact that the measurements were conducted immediately after completion of the anastomosis. Although an arterial pulse was present, as detected by the pulse oximeter, and a haemoglobin signal was measured by the MSI system, the full extent of the microcirculation may not have been reestablished at that point.

The pulse oximeter gave consistently higher values of oxygen saturation than MSI for each measurement in both animals. The most likely explanation for this is that the pulse oximeter uses the arterial pulse as a 'biological filter' to isolate the absorption signal of arterial vessels from background attenuation. The MSI system measures both venous and arterial components (including the parenchyma), which could be expected therefore to be much lower, in some cases, than the arterial component. Furthermore it is possible that the equal pathlength assumption built into the use of Eq. 1 is insufficient. Light towards the red end of the wavelength range used penetrates deeper than that at the blue end and therefore interrogates a larger tissue volume. If this pathlength difference is significant then it could result in a higher apparent absorbance at the red end and errors in $\mathrm{SaO}_{2}$ calculation.

\section{CONCLUSIONS}

A multispectral imaging laparoscope has been tested as a method for monitoring tissue oxygenation during uterine transplantation surgery in a rabbit and a sheep, and shown to display similar trends in oxygenation as that reported by pulse oximetry.

MSI proved to be a convenient method as it was contact-free and, unlike pulse oximetry, required no complex manipulation of the tissue. It also has the advantage of being an imaging technique giving the surgeon information with high spatial resolution on all visible areas of the tissue. This contrasts with pulse oximetry, which is limited to single point measurements.

Future work on the MSI hardware will focus on improving the system speed. Data reduction techniques will be used to minimise the number of wavelengths needed in each acquisition, while implementation of the $\mathrm{SaO}_{2}$ regression algorithm on the GPU will provide massive parallelisation. Coupled with the fact that the registration algorithm can already converge within the millisecond range, true real-time operation is possible. In addition to this, further analysis of the oxygenation data from both measurement techniques over several surgical trials for each animal is needed to fully quantify intra- and inter-subject variability, and investigate the relationship between the pulse oximetry and MSI output.

\section{ACKNOWLEDGEMENTS}

Funding for this work was provided by ERC grant 242991, UK EPSRC grants EP/E06342X/1 and DT/E011101/1, and Uterine Transplant UK Charity (number 1138559). Neil Clancy would like to gratefully acknowledge the financial support of an Imperial College Junior Research Fellowship. Danail Stoyanov is supported by a Royal Academy of Engineering/EPSRC Fellowship. The authors would like to thank the Royal Veterinary College for their assistance with surgical arrangements.

\section{REFERENCES}

[1] Matsui, A., Winer, J. H., Laurence, R. G. and Frangioni, J. V., "Predicting the survival of experimental ischaemic small bowel using intraoperative near-infrared fluorescence angiography," Br. J. Surg. 98(12), 1725-1734 (2011).

[2] Sowa, M. G., Payette, J. R., Hewko, M. D. and Mantsch, H. H., "Visible-near infrared multispectral imaging of the rat dorsal skin flap," J. Biomed. Opt. 4(4), 474-481 (1999). 
[3] Ilias, M. A., Häggblad, E., Anderson, C. and Salerud, E. G., "Visible, hyperspectral imaging evaluating the cutaneous response to ultraviolet radiation," Proc. SPIE 6441, 644103 (2007).

[4] Sorg, B. S., Moeller, B. J., Donovan, O., Cao, Y. and Dewhirst, M. W., "Hyperspectral imaging of hemoglobin saturation in tumor microvasculature and tumor hypoxia development," J. Biomed. Opt. 10(4), 044004 (2005).

[5] Nighswander-Rempel, S. P., Shaw, R. A., Mansfield, J. R., Hewko, M., Kupriyanov, V. V. and Mantsch, H. H., "Regional variations in myocardial tissue oxygenation mapped by near-infrared spectroscopic imaging," J. Mol. Cell. Cardiol. 34(9), 1195-1203 (2002).

[6] Bryan, R. T., Billingham, L. J. and Wallace, D. M. A., "Narrow-band imaging flexible cystoscopy in the detection of recurrent urothelial cancer of the bladder," Br. J. Urol. 101(6), 702-706 (2007).

[7] Takano, J. H., Yakushiji, T., Kamiyama, I., Nomura, T., Katakura, A., Takano, N. and Shibahara, T., "Detecting early oral cancer: narrowband imaging system observation of the oral mucosa microvasculature," Int. J. Oral Maxillofac. Surg. 39(3), 208-213 (2010).

[8] Wolfsen, H. C., Crook, J. E., Krishna, M., Achem, S. R., Devault, K. R., Bouras, E. P., Loeb, D. S., Stark, M. E., Woodward, T. A., Hemminger, L. L., Cayer, F. K. and Wallace, M. B., "Prospective, controlled tandem endoscopy study of narrow band imaging for dysplasia detection in Barrett's esophagus," Gastroenterology 135(1), 24-31 (2008).

[9] Zuzak, K. J., Gladwin, M. T., Cannon, R. O. I. and Levin, I. W., "Imaging hemoglobin oxygen saturation in sickle cell disease patients using noninvasive visible reflectance hyperspectral techniques: effects of nitric oxide," Am. J. Physiol. Heart Circ. Physiol. 285(3), H1183-H1189 (2003).

[10]Ramella-Roman, J. C. and Mathews, S. A., "Spectroscopic measurements of oxygen saturation in the retina," IEEE Sel. Top. Quantum Electron. 13(6), 1697-1709 (2007).

[11]Stoyanov, D., Rayshubskiy, A. and Hillman, E., "Robust registration of multispectral images of the cortical surface in neurosurgery," IEEE ISBI, 1643-1646 (2012).

[12]Tracy, C. R., Terrell, J. D., Francis, R. P., Wehner, E. F., Smith, J., Litorja, M., Hawkins, D. L., Pearle, M. S., Cadeddu, J. A. and Zuzak, K. J., "Characterisation of renal ischemia using DLP hyperspectral imaging: a pilot study comparing artery-only occlusion versus artery and vein occlusion," J. Endourol. 24(3), 321-325 (2010).

[13]Crane, N. J., Huffman, S. W., Alemozaffar, M., Gage, F. A., Levin, I. W. and Elster, E. A., "Evidence of a heterogeneous tissue oxygenation: renal ischemia/reperfusion injury in a large animal model," J. Biomed. Opt. 18(3), 035001 (2013).

[14]Clancy, N. T., Sauvage, V., Saso, S., Stoyanov, D., Corless, D. J., Boyd, M., Noakes, D. E., Yang, G.-Z., GhaemMaghami, S., Smith, J. R. and Elson, D. S., "Registration and analysis of multispectral images acquired during uterine transplantation surgery," OSA Technical Digest, BSu3A.73 (2012).

[15]Del Priore, G., Schlatt, S., Wagner, R., Reynoso, E. and Malanowska-Stega, J., "Uterus transplantation: on the edge," Semin. Reprod. Med. 29(1), 55-60 (2011).

[16]Brännström, M., Wranning, C. A. and Altchek, A., "Experimental uterus transplantation," Hum. Reprod. Update $16(3), 329-345$ (2010).

[17]Brännström, M., Diaz-Garcia, C., Hanafy, A., Olausson, M. and Tzakis, A., "Uterus transplantation: animal research and human possibilities," Fertil. Steril. 97(6), 1269-1276 (2012).

[18]Moxey, P., Sieunarine, K., Cox, J., Lawson, A. D., Ungar, L. and Smith, J. R., "Pulse oximetry and perfusion index measurement to assess uterine perfusion and viability," Int. Surg. 91(4), 223-227 (2006).

[19]Prahl, S. A., "Tabulated molar extinction coefficient for hemoglobin in water," OMLC, 4 March 1998, (June 2009). http://omlc.ogi.edu/spectra/hemoglobin/summary.html

[20]Clancy, N. T., Stoyanov, D., James, D. R. C., Di Marco, A., Sauvage, V., Clark, J., Yang, G.-Z. and Elson, D. S., "Multispectral image alignment using a three channel endoscope in vivo during minimally invasive surgery,"

Biomed. Opt. Express 3(10), 2567-2578 (2012). 